\title{
GRG Update: DDW 2011
}

\author{
Rhonda F. Souza
}

Published online: 5 August 2011

(C) Springer Science+Business Media, LLC 2011 (Outside the USA) 2011

\section{GRG Spring Symposium}

I would like to take this opportunity to update the readership of Digestive Diseases and Sciences (DDS), including members of the GRG, on the 2011 GRG Spring Symposium. DDW was held at McCormick Place in Chicago, and followed the "new" 4-day format. In doing so, the GRG Symposium was moved to Monday morning rather than its traditional Sunday afternoon slot in order to avoid time conflicts with other basic science-oriented sessions. This year's symposium, entitled "MicroRNAs as Pleiotropic Regulators in Digestive and Liver Disease," was chaired by Dr. Nick Davidson, Washington University, St. Louis, and myself. The following outstanding state-of-the-art lectures were presented: Fractal Genetics and Systems Biology of Diet-Induced Metabolic Diseases by Dr. Joseph Nadeau, Boston University; MicroRNAs as Regulators of Hepatocyte Proliferation and Regeneration by Dr. Holger Willenbring, University of California San Francisco; and MicroRNAs as Regulators of Intestinal Inflammation by Dr. Didier Merlin, Emory University.

\section{GRG/AGA Young Investigator Awards}

The central mission of today's GRG is to foster, support, and promote the careers of young investigators (physiciansscientists and doctoral scientists) in digestive and liver disease research. One way in which the GRG continues to achieve this mission is through recognizing the exceptional

\section{R. F. Souza $(\square)$}

Department of Medicine, VA North Texas Health Care

System-Dallas and University of Texas Southwestern Medical

Center, Dallas, TX, USA

e-mail: rhonda.souza@utsouthwestern.edu achievements of investigators conducting digestive disease research with the GRG/AGA Young Investigator Awards for Basic and Clinical Science Research.

\section{Young Investigator Award for Clinical Science Research:}

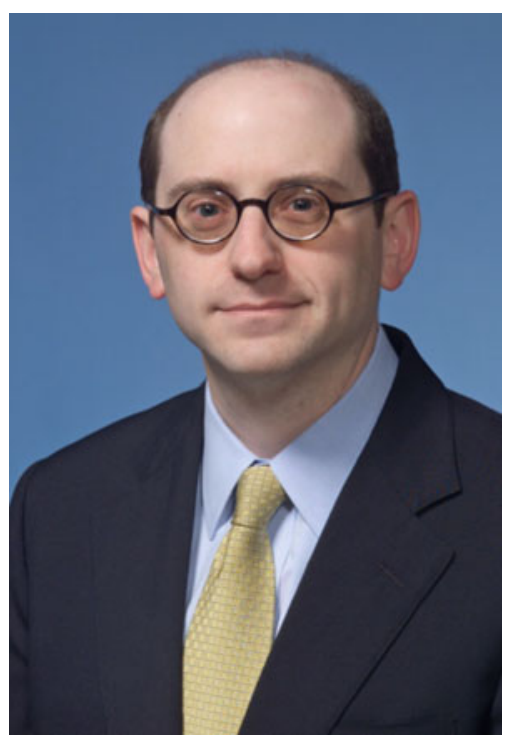

Joel Rubenstein earned his undergraduate degree with Highest Distinction from the University of Michigan. He attended the medical school at Washington University in St. Louis and completed his residency training at the University of Chicago. Dr. Rubenstein received his gastroenterology training at the University of Michigan and, whilst there, earned a Masters Degree in Clinical Research Design and Statistical Analysis. He is currently an Assistant Professor at the University of Michigan, a staff physician at the Ann 
Arbor VA, and a Research Scientist at the VA Center for Clinical Management Research. Dr. Rubenstein's research is focused on the epidemiology and outcomes in Barrett's esophagus and esophageal adenocarcinoma. He has published 30 peer-reviewed articles and has received research funding from multiple sources, including the NIH and the Damon Runyon Cancer Research Foundation Gordon Family Clinical Investigatorship.

\section{Young Investigator Award for Basic Science Research:}

\section{Noah F. Shroyer, Ph.D.}

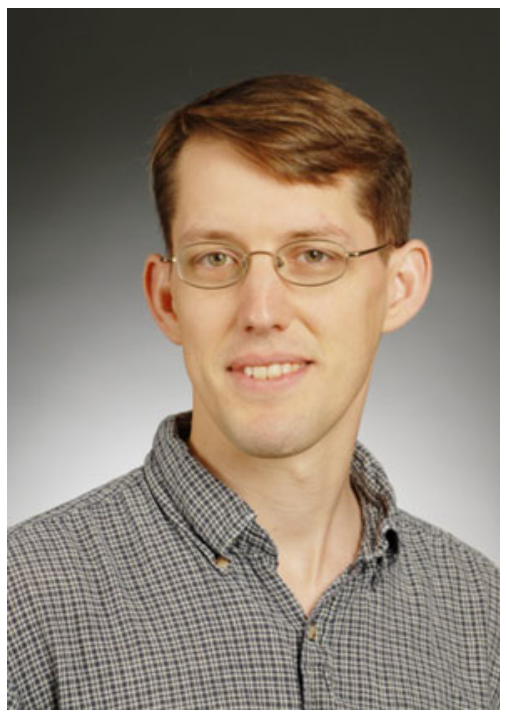

Dr. Shroyer graduated from Louisiana State University with a B.S. in Biochemistry and Microbiology. He received his Ph.D. in Cell and Molecular Biology and completed postdoctoral training in Molecular and Human Genetics at Baylor College of Medicine, Houston, TX. He is currently an Assistant Professor in the Section of Gastroenterology, Hepatology, and Nutrition, Department of Pediatrics, as well as in the Division of Developmental Biology at Cincinnati Children's Hospital Medical Center. Dr. Shroyer's research is focused on understanding the mechanisms that control intestinal development and homeostasis, and translating this knowledge into novel therapeutic approaches to treat diseases of the intestine, such as colorectal cancer. His laboratory has elucidated roles for epithelial transcription factors such as Atoh1 (Math1), Gfi1, and Spdef in the development and differentiation of the intestine. He has published over 30 original peer-reviewed publications and has been awarded three research grants, including an NIH R01 to study mechanisms of colorectal carcinogenesis.
GRG Fellow Travel Co-Abstracts of the Year: Taku Kobayashi, M.D., Ph.D. and Maximilian Reichert, M.D.

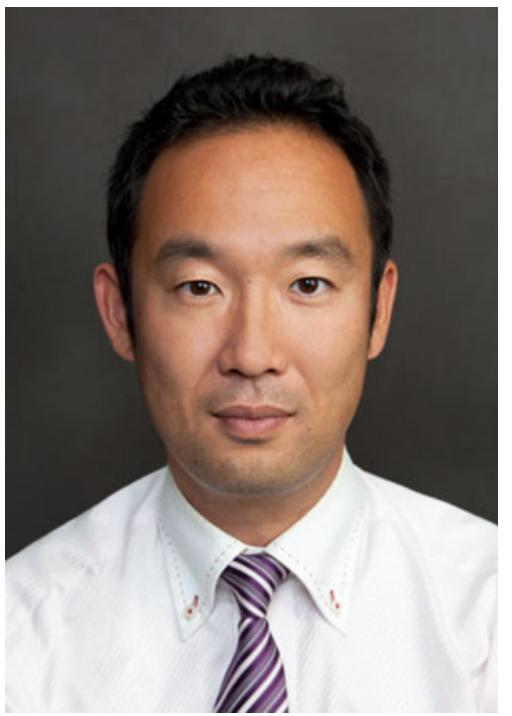

Dr. Kobayashi obtained his MD from Nagoya University School of Medicine and his Ph.D. from Nagoya University Graduate School of Medicine, Nagoya, Japan. Dr. Kobayashi's residency in internal medicine was completed at Toyohashi Municipal Hospital, Aichi, Japan, and his clinical gastroenterology fellowship was completed at the KKR Tokai Hospital, Nagoya. Dr. Kobayashi also completed a gastroenterology research fellowship at Keio University, Tokyo, Japan. He is currently a postdoctoral fellow in the Division of Gastroenterology at the University of North Carolina. Under the mentorship of Dr. Scott Plevy, Dr. Kobayashi's work is focusing on the role of the innate inflammatory response in mucosal immunity as it relates to inflammatory bowel disease.

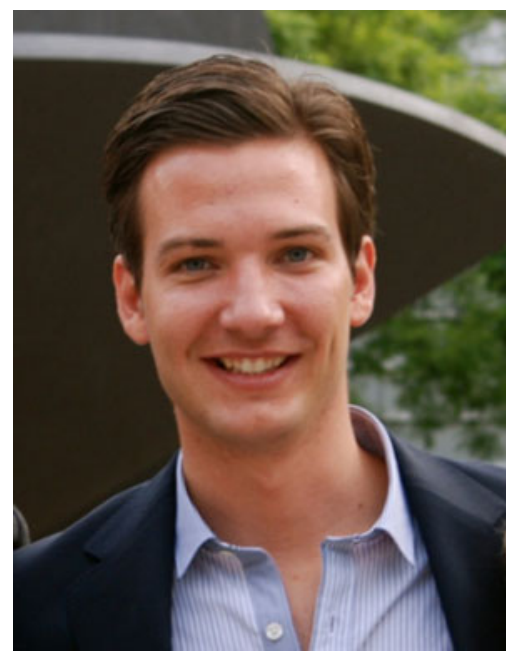


Dr. Reichert undertook his MD training at the RuprechtKarls University of Heidelberg and Technical University of Munich Medical Schools in Germany. Dr. Reichert's residency in internal medicine and gastroenterology was completed at the Technical University of Munich. He is currently a postdoctoral fellow in the Division of Gastroenterology at the University of Pennsylvania. Under the mentorship of Dr. Anil Rustgi, Dr. Reichert's work is focusing on the molecular regulation of pancreatic ductal epithelial morphogenesis.

\section{Final Words}

Planning for the 2012 GRG Spring Symposium is currently underway. Our moderators will be Dr. William Grady, our new GRG President-Elect, and Dr. Andrew Chan. For information about the GRG Awards and other GRG activities, please visit our website: http://www.gastro research.org.

I also encourage members of the GRG to submit original clinical and basic science manuscripts to $D D S$. Finally, I also encourage the GRG's senior mentors to continue to work with our young investigators by encouraging the submission of up-to-date translational research reviews to $D D S$ as a means for the GRG to advance its mission to "foster, support, and promote the careers of young investigators" in digestive disease research. 